\title{
ATTITUDE ASSESSMENT USING PLEIADES-HR CAPABILITIES
}

\author{
Jean-Marc DELVIT ${ }^{\mathrm{a}}$, Daniel GRESLOU ${ }^{\mathrm{a}}$, Virginie AMBERG ${ }^{\mathrm{a}}$, Cécile DECHOZ $^{\mathrm{a}}$, Françoise DELUSSY ${ }^{\mathrm{a}}$, Laurent LEBEGUE $^{\mathrm{a}}$, \\ Christophe LATRY ${ }^{\mathrm{a}}$, Stéphanie ARTIGUES ${ }^{\mathrm{b}}$, Laurent BERNARD ${ }^{\mathrm{b}}$ \\ ${ }^{a}$ CNES, 18, avenue Edouard Belin 31401 TOULOUSE CEDEX 4 - jean-marc.delvit@cnes.fr \\ ${ }^{\mathrm{b}}$ Magellium, 1824 rue Hermès, BP 12113, 31521 Ramonville Saint-Agne, France
}

\section{Commission VI, WG VI/4}

KEY WORDS: calibration, image quality, geometry, aerial triangulation, elevation model, Pléiades-HR

\begin{abstract}
:
Since SPOT1, the French national space centre (CNES) has worked on improving the geometry of Earth observation spacecrafts. The accuracy of sensor calibration is one of the main key points for any Earth observation application such as orthorectification, DEM generation or surface change detection. For the last twenty years CNES has developed two families of methods: absolute methods and relative methods. These methods are used to characterize a pushbroom acquisition along the detector line and the time line. By this way, the viewing directions are measured and the residual of the spacecraft's attitude angles (not restituted by the Attitude and Orbit Control System) is estimated. This information can complete the geometric model of all the scenes acquired by the instrument and is used in all geometric applications. This paper presents new attitude assessment methods taking advantage of the capabilities of Pléiades-HR in terms of agility and focal plane arrangement - panchromatic band and multispectral (MS) bands.
\end{abstract}

\section{INTRODUCTION}

Since SPOT1, the French national space centre (CNES) has worked on improving the geometry of Earth observation spacecrafts. The accuracy of sensor calibration is one of the main key points for any Earth observation application such as orthorectification, DEM generation or surface change detection. For the last twenty years CNES has developed two families of methods: absolute methods and relative methods. These methods are used to characterize a pushbroom acquisition along the detector line and the time line. By this way, the viewing directions are measured and the residual of the spacecraft's attitude angles (not restituted by the Attitude and Orbit Control System) is estimated. This information can complete the geometric model of all the scenes acquired by the instrument and is used in all geometric applications. This paper presents new attitude assessment methods taking advantage of the capabilities of Pleiades-HR in terms of agility and focal plane arrangement - panchromatic band and multispectral (MS) bands. This paper also presents new method to calibrate static geometry in terms of biases and viewing directions.

\section{PLEIADES-HR}

PLEIADES-HR is the highest resolution civilian earth observing system ever developed in Europe. This imagery program is conducted by the French National Space Agency, CNES. It is the French part of the French-Italian ORFEO program which also comprises COSMO-SkyMed, an Italian high-resolution radar system. It will operate in 2011-2012 two agile satellites designed to provide optical images to civilian and defense users. Images will be simultaneously acquired in Panchromatic (PA) and multi-spectral (MS) mode, which allows, in nadir acquisition condition, to deliver $20 \mathrm{~km}$ wide, false or natural colored scenes with a $70 \mathrm{~cm}$ ground sampling distance after PA+MS fusion. Coverage will be almost worldwide with a revisit interval of $24 \mathrm{~h}$ for 2 satellites.

The Image Quality requirements were defined from users' studies from the different spatial imaging applications, taking into account the trade-off between on-board technological complexity and ground processing capacity. The PLEIADESHR satellites will benefit from technology improvements in various fields which will allow achieving, at an affordable price, performances once reserved to ambitious military spacecrafts.

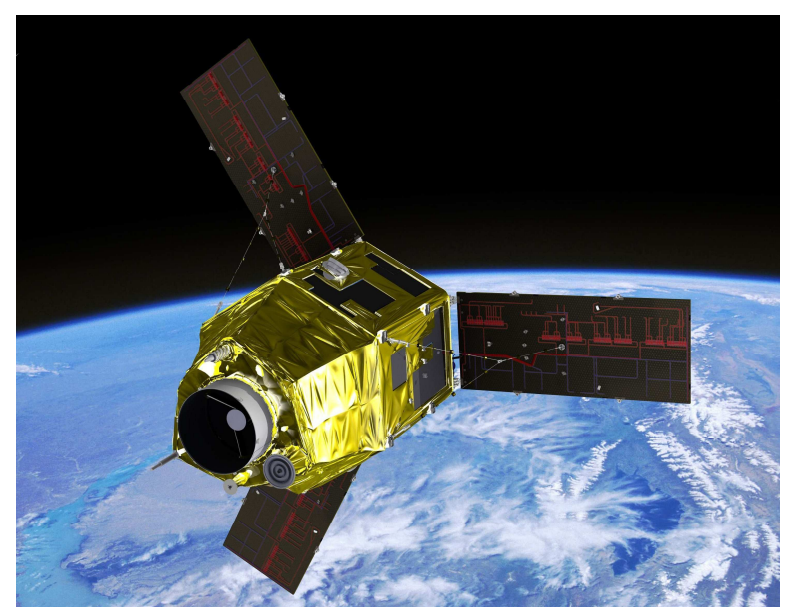

Figure 1: the Pleiades-HR satellite

The major constraints of weight and agility led to the development of a highly compact satellite (about 1 ton weight), to minimize the moments of inertia. The instrument is partly embedded in a hexagonal shaped bus containing all equipment. The attitude control system uses 4 fiber-optic gyroscopes and 3 star trackers to provide good restitution accuracy. Furthermore, 
these attitude sensors are mechanically fixed on the telescope support to minimize the thermo-elastic distortions in order to be compatible with the system specifications.

Agility is a characteristic which allows the satellite to acquire off-nadir targets rapidly in a large flight envelope, in order to sequence numerous images. This agility is imposed by several requirements stated by the users. For instance, a 100x100 km2 zone can be acquired by the satellite from the same orbit thanks to a lateral multi-band coverage. As for stereoscopic capacities, 3 images from the same zone can be acquired in a single pass with $\mathrm{B} / \mathrm{H}$ lying between 0.1 and 0.5 . For multi-targets, the time between the end of an imaging segment and the start of the next segment, including stabilization of the line-of-sight is specified less than 10 seconds for an excursion of $10^{\circ}$ and less than 26 seconds for an excursion of $60^{\circ}$ from nadir viewing. Guidance is mainly performed using roll and/or pitch steering (without slow motion), but fine yaw steering has to be used to respect the principle of acquisition set by the TDI device in the focal plane [9], [13]. This agility can be used to calibrate the whole system [14]!

\section{BIASES AND ABSOLUTE LOCATION ASSESSMENT}

\subsection{Classical method using GCPs}

The system's absolute location error stands for the deviation between the real ground position of any point of the image and its estimated position using the image geometrical model [3]. The method used to estimate the absolute location accuracy is based on a physical modeling of the acquisition and the use of very accurate ground control points, called GCPs. Using the same GCPs, the estimation of the biases is performed with a block adjustment by space triangulation method [12]. Some geographic areas are very well known including GPS points or aerial images and 3d models (Figure 2).

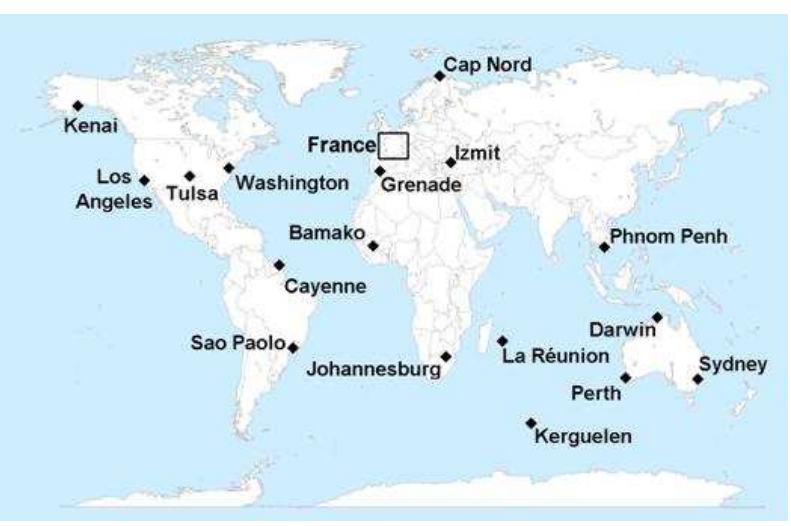

Figure 2: biases assessment using well known geographic sites

\subsection{A new method using Pléaides-HR capabilities}

Pleiades allows making reverse acquisitions. These acquisitions are obtained on the same site with a $180^{\circ}$ difference of yaw. Thanks to these acquisitions, an unknown roll or/and pitch can be interpreted in a direction in the first image and in the opposite direction in the second image. The biases in roll and pitch are statistically zero, the difference between reverse acquisitions are the statistically equal to the double of the biases.

The method used to assess the biases can be decomposed in 3 steps (Figure 3).

1: For a reverse acquisition, we choose a reference. The second image is then resampled in the geometry of the reference using the geometric model. The two images are nearly superimposable. The only differences are due to the lack of knowledge of the alignment biases.

2: The second step of this method consists in correlating the reference image and the resampled image, in order to find tie points between the two products. The mean difference along the row is caused by two pitch biases (one for each product), and the mean difference along the column is caused by two roll biases (one for each product). These biases are estimated with a block adjustment method using all the tie points computed.

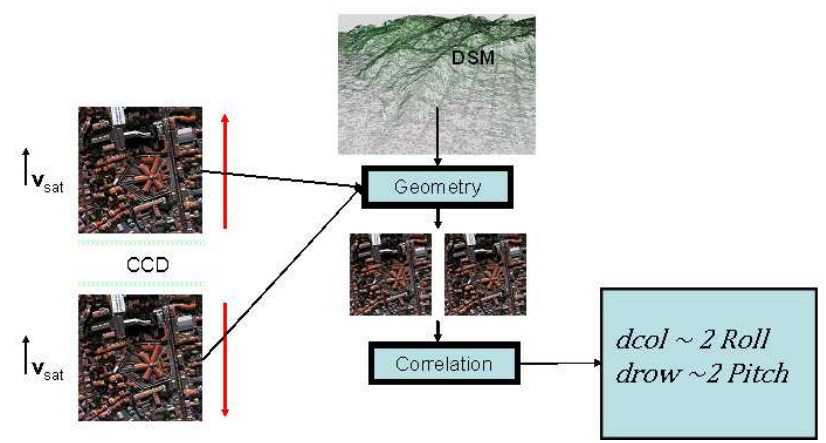

Figure 3: bias assessment using reverse acquisitions

3: the biases can be statistically estimated using a lot of reverse acquisitions. The distribution mean is linked to the roll and pitch biases, and the standard deviation is correlated to the location performance.

The use of cycled couples (images acquired every 26 days in the same conditions) is needed to avoid all the stereoscopic effects if the digital elevation model is unknown.

We can also note that in step 2 if we reverse the reverse acquisitions (changing the reference), we obtain the same results.

\section{Some Pleiades-HR results}

Reverse acquisitions allow assessing absolute location without absolute references. It is possible to use geographic sites in high latitudes.

These acquisitions were used successfully in Pleiades-HR to find the first coarse alignment biases.

This method was also qualified on GPS geographic sites. The biases assessment has been compared using the classical method and the cycled reverse acquisitions. The results show a difference of less than $2 \mu \mathrm{rad}$. This error is compatible with the correlation accuracy for the new method and the accuracy of the manual GPS pointing for the classical one. 


\section{VIEWING DIRECTIONS MEASURMENT}

\subsection{Classical method using a geometric supersite}

The cartography of the focal plane can also use a dedicated reference site [1], called geometric supersite, covered by many high-resolution aerial images which are very accurately georeferenced thanks to ground control points and fine DEM (Figure 3). When the satellite overpasses the site, an image is acquired and then compared to the reference site projected in the focal plane geometry (Figure 4). This approach requires a cost-effective update of the aerial imagery and an uphill geometric work. Moreover, the quality of the reference is linked to the season of the acquisition of the supersite. For example, the Supersite of Salon de Provence in France (Figure 5) offers two sets of acquisitions- one in March and the other in September [5]. This kind of reference can also be used to characterize the residual of the spacecraft's attitude angles (not restituted by the AOCS) but not to improve the attitude.
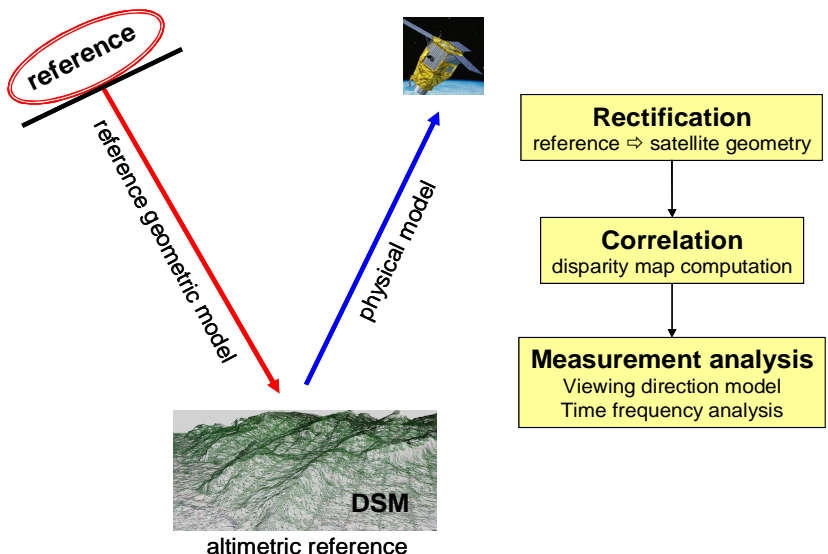

Figure 4: Description of the method

Other kinds of references can also be used: spatial high resolution images can be used instead of aerial images [2], [4]. The ground sampling distance ratio between the reference and the images must be sufficiently low, in order to consider the reference as perfect [6], [7]. The most difficult is to have a $3 \mathrm{~d}$ reference which can be produced with stereoscopic spatial data.

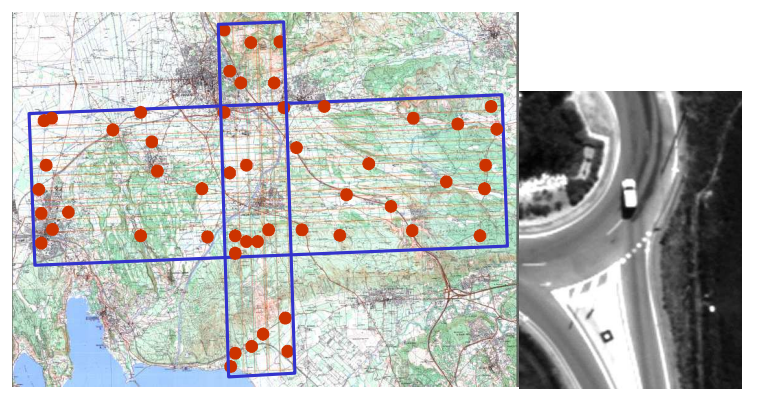

Figure 5: the geometric supersite of Salon de Provence composed of 52 GPS GCPs, 1149 aerial images and their accurate geometric model, and a $1 \mathrm{~m}$ DSM.

\subsection{A new method using a Pléaides-HR capabilities}

A new method, called geometric auto-calibration, will be applied to achieve this cartography without any reference site. The idea is to acquire the same site at least twice on the same orbit thanks to the satellite agility, with track orientations close to orthogonality (Figure 6).

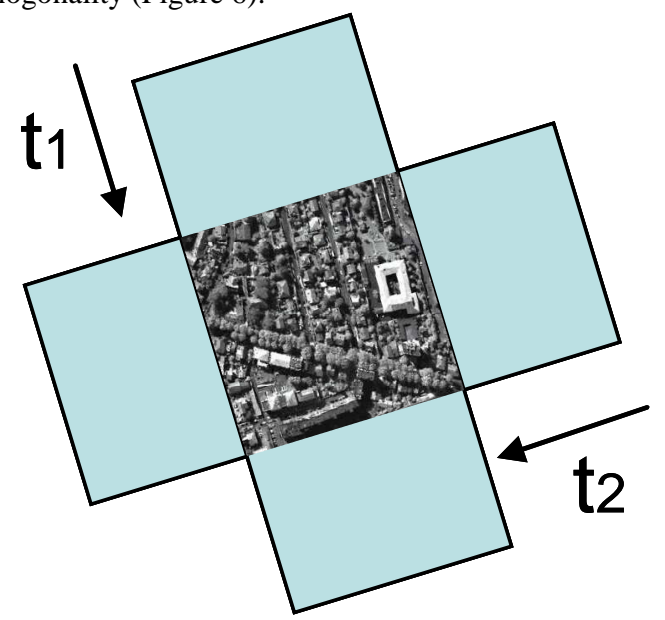

Figure 6: cross-track acquisitions of a same landscape

The two images obtained could be derived from each other with a coarse $90^{\circ}$ rotation. In practice, one is re-sampled into the other one's geometry thanks to the available accurate geometric models and a digital elevation model as shown on Figure 1. Sites without lots of $3 \mathrm{~d}$ objects can be used. The new images can be overlaid but include opposite column-wise and line-wise behavior according to temporal attitude effects and CCD geometry (Figure 7).

The two images are correlated and statistical computation is applied on the lines and columns of the disparity map. One direction gives information on the lack of knowledge of the focal plane's cartography and on the attitude high frequency residues not measured by the AOCS for the " $0^{\circ}$ image". The other direction gives information on the lack of knowledge of the same focal plane's cartography and on the attitude high frequency residues not measured by the AOCS for the " $90^{\circ}$ image".
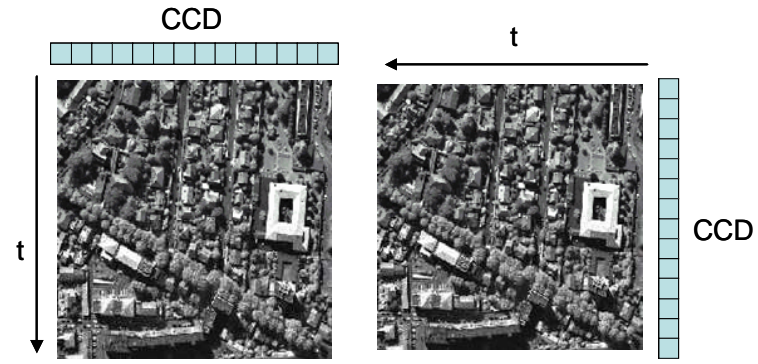

Figure 7: images obtained from geometric auto-calibration.

A simple frequency filter (depending on the cutoff frequency of the AOCS) can separate the information of the cartography from the attitude information. Then, the focal plane cartography can be modelized by a polynomial.

The autocalibration method allows estimation of the focal plane cartography with an accuracy of 0.03 pixel RMS for simulations. The characterization of the attitude high frequency residues not measured by the AOCS is correctly done and perturbation frequencies are found for the two images. 


\section{Some Pleiades-HR results}

At first this method can be used to assess the yaw biases with accuracy. In fact the slope of the estimation along the row is directly the yaw (Figure 8).

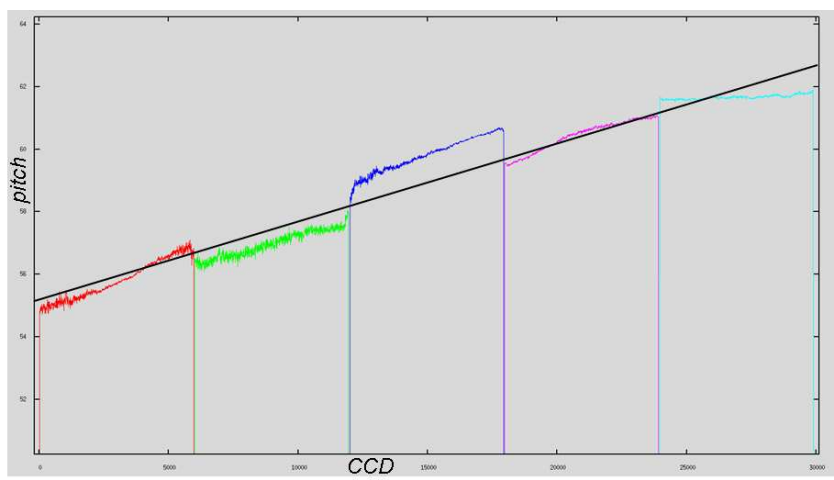

Figure 8: yaw estimation using cross acquisitions

Once the linear part of the model has been withdrawn, the residuals seen in Figure 9 show the viewing directions in the two directions (row wise and column wise). The Figure 10 shows the viewing directions for a Pleiades CCD array assessed with the cross acquisition (red line with a noise less than $0.05 \mu \mathrm{rad} \max$ ) and the supersite (blue line with a noise less than $0.15 \mu \mathrm{rad} \max )$. The inter-array overlapping is also measured in order to minimize geometric default between two successive detectors.
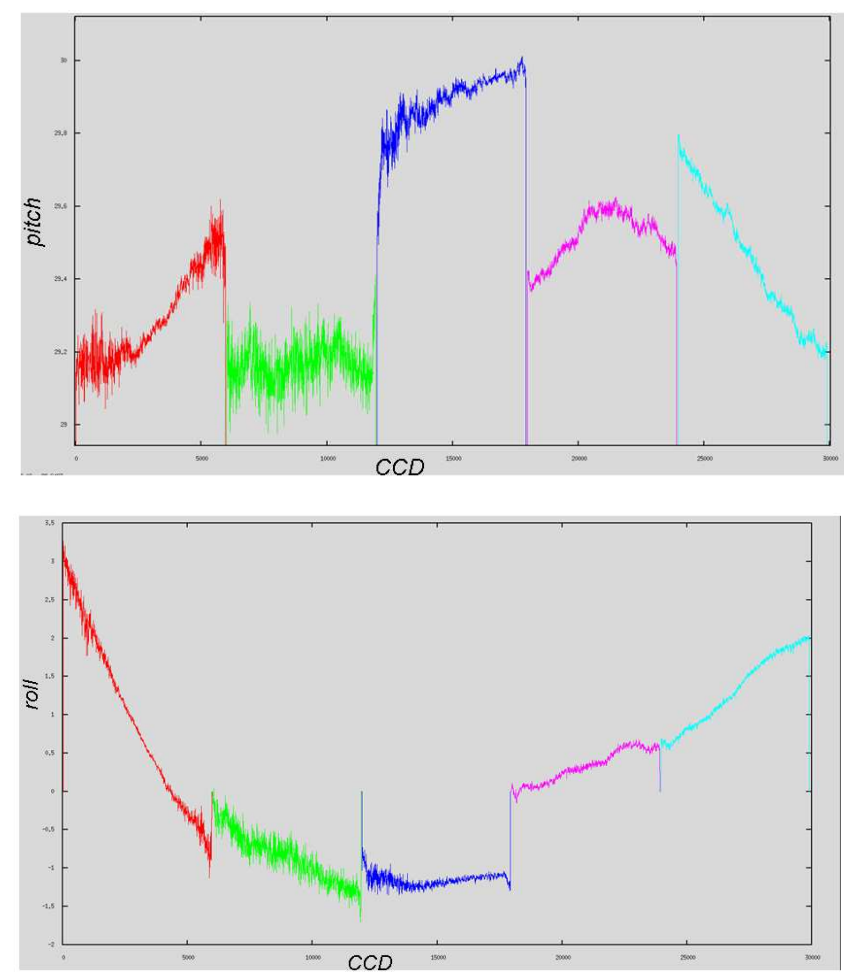

Figure 9: viewing directions measurement using cross acquisition

If the altitude of the scene is unknown, the use of cycled orthogonal couples (images acquired every 26 days in the same conditions) is needed to avoid all the stereoscopic effects.

\section{ATTITUDE ASSESSEMENT}

Another way to improve the geometric model is to use the focal plane abilities in order to improve the residual of the spacecraft's attitude angles (not restituted by the AOCS). For example in Figure 11, the Pléiades-HR MS focal plane can be used to overpass the AOCS restitution frequency [8].

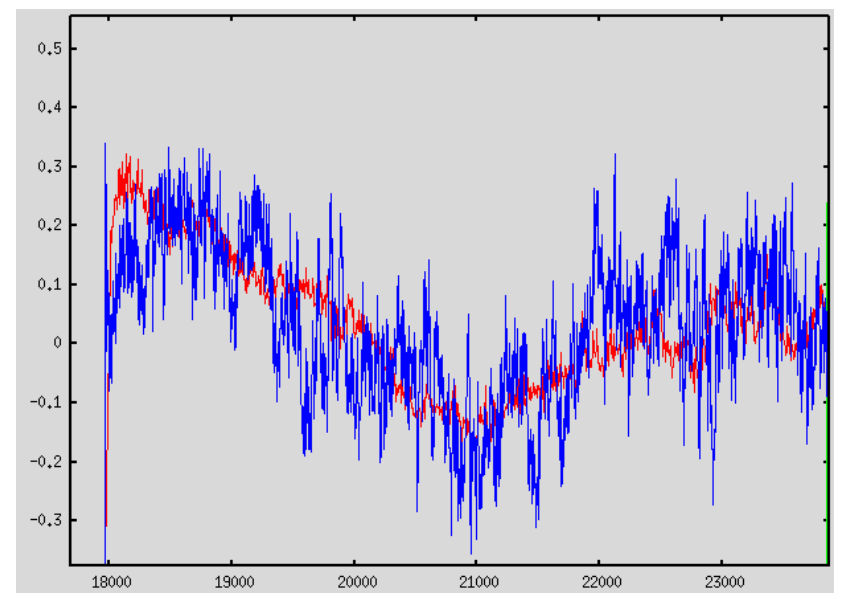

Figure 10: viewing directions measurement using cross acquisition (red) and supersite (blue). The cross acquisition is less noisy than the classical method.

For Pleiades-HR focal plane the panchromatic/multispectral shift is significant: $19 \mathrm{~mm}$ in the focal plane, which means $1 \mathrm{~km}$ on ground, a time delay of $0.15 \mathrm{~s}$ and a $1.5 \mathrm{mrad}$ stereoscopic angle. Maximum offset between two multispectral bands is 6 times smaller ( $3 \mathrm{~mm}$ max).

A ground point is successively acquired in four spectral bands. The four images are radiometrically correlated. Because of the pushbroom concept, one image line is associated to a time $t$ and the mean disparity column gives insight to the differential perturbation $f(t+d t)-f(t)$, with dt being the time delay that ranges between the two acquisitions of a given ground point by the two CCDs. The correlation process between these two CCDs allows to measure very low amplitude perturbations. The dt time delay, linked to the physical offset of the staggered arrays, is a key figure for determining the frequency range of this measurement tool.

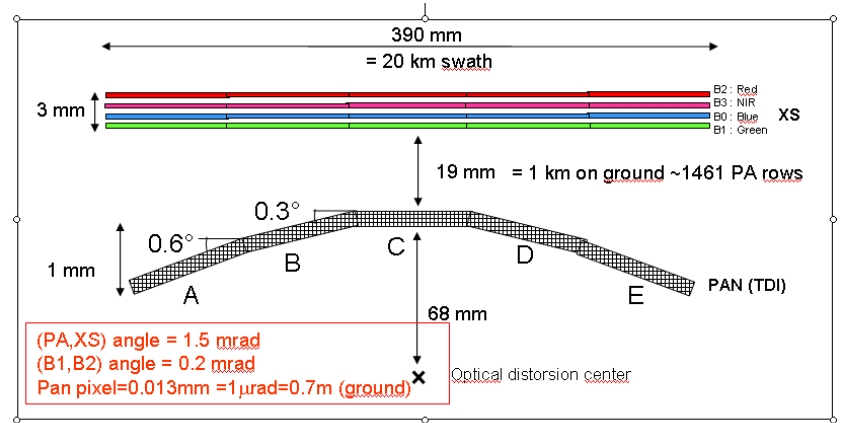

Figure 11: Pleiades-HR staggered focal plane.

If the perturbation frequency is correlated with the distance separating the two MS bands, it is possible to use the panchromatic tilt. In this case the MS is used as an intermediate in order to access to a weak time difference ( $\tau^{\prime}$ in Figure 12). 


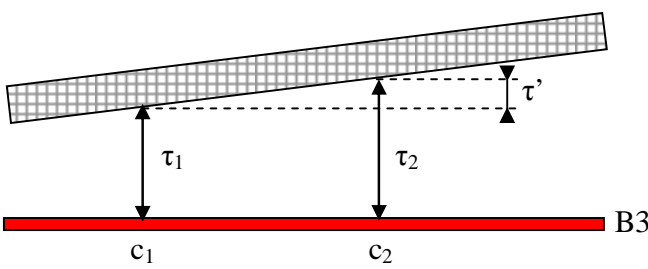

PAN

Figure 12: panchromatic tilt use

A hybridization using the MS method and the tilt globally improves the restitution.

If the focal plane is unknown, the correlation can also be performed within the image. In fact, the differential perturbation $\mathrm{f}(\mathrm{t}+\mathrm{dt})-\mathrm{f}(\mathrm{t})$ can be obtained by the correlation of two following lines, supposing that the landscape is stable. In this case, a Wiener integration can estimate the perturbation $f(t)$, improve the geometric modelization and therefore the geometric image quality. This method is limited by construction to roll perturbations (along the CCD), but is able to estimate noisy roll perturbation.

\section{Some Pleiades-HR results}

Favorable case:

In a complete Pleiades simulation (without critical frequencies) case, the method using the correlation between the MS and the panchromatic tilt improve the attitude assessment up to $10 \%$ (green curve in Figure 13) comparing to the MS only method (blue curve in Figure 13). The hybrid method combines the advantages of the two previous methods. No frequency holes are present and the performances are better: this method improves the attitude assessment up to $22 \%$ in this case.

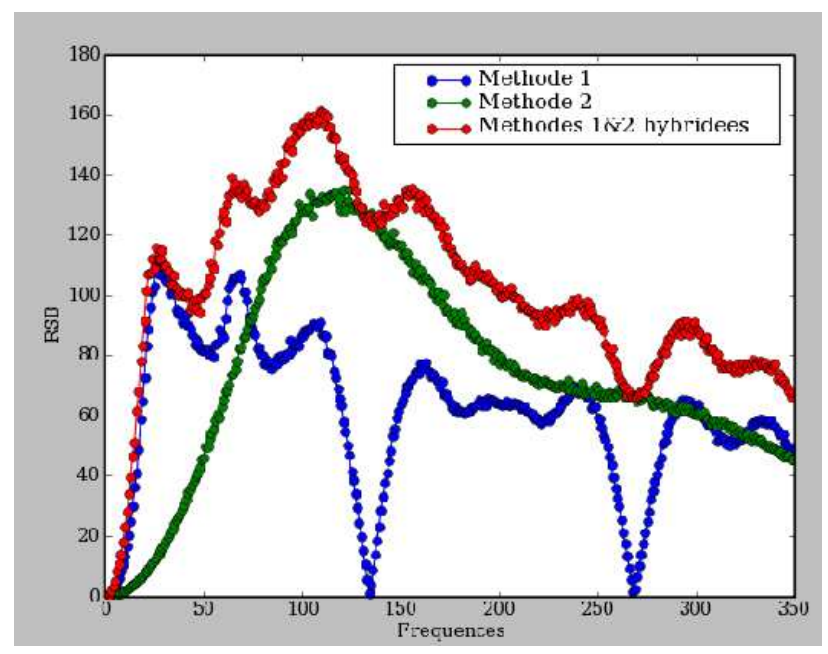

Figure 13: performance of restitution using MS correlation only (blue), tilt only (green), and MS and tilt correlation (red)

Difficult case:

If we consider a simulation with critical frequencies (near the frequency holes of the classical MS method), the method using the correlation between the MS and the panchromatic tilt improve the attitude assessment up to $25 \%$ (Figure 14) comparing to the MS only method (Figure 14). The hybrid method combines the advantage of the two previous methods. No frequency holes are present and the performances are better: this method improves the attitude assessment up to $50 \%$ in this case.

These three methods are tested on real data [11]. The first results reveal that all the predicted frequencies on the ground are present in the flight. Yet, the amplitudes of these signals are very weak (less than $0.1 \mu \mathrm{rad}$ ). Results have to be confirmed.
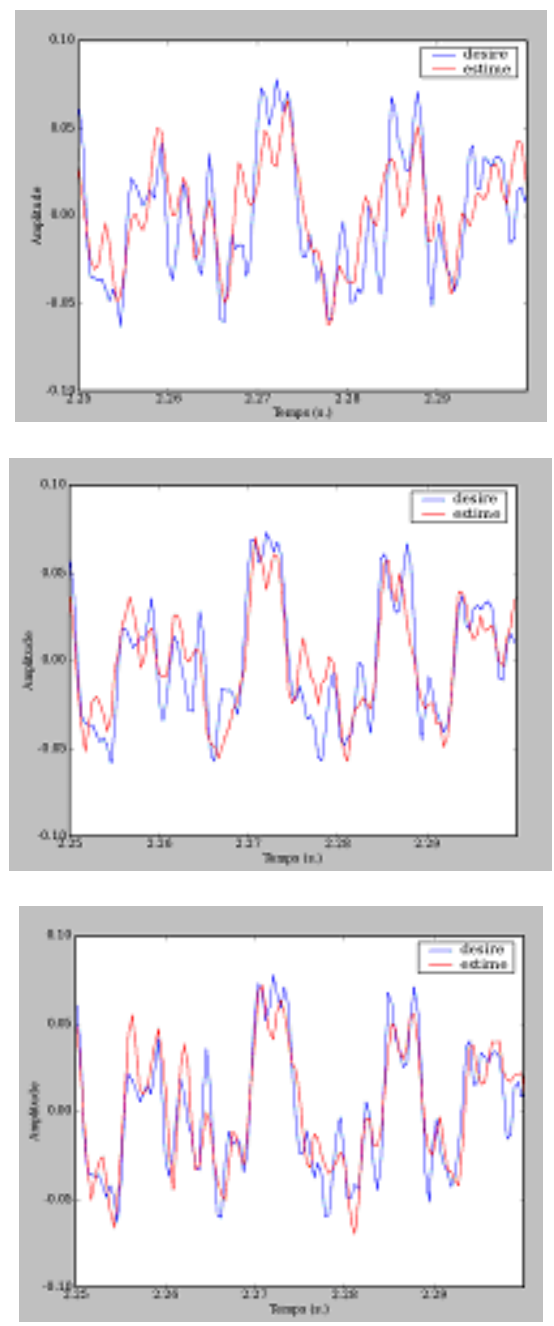

Figure 14: example of restitution using MS correlation only (up scheme), tilt only (middle scheme), and MS and tilt correlation (bottom scheme) - in blue the signal to assess; in red the signal assessed. 


\section{CONCLUSION}

All these attitude assessment methods are useful to understand the system and to improve the auxiliary data of products acquired by the system, especially data necessary to build the geometric model. These improvements allow registration accuracy better than 0.1 pixel, which is a requirement for most Earth observation applications (3d reconstruction, $2 \mathrm{~d}$ and $3 \mathrm{~d}$ change detection, pan sharpening, image mining...).

New methods of calibration appear with the agility of new satellites. These methods neither need specific views like supersite for geometric calibration nor external geometric references. This is a real advantage for such satellite and this is really appreciated by users and commercial providers. The calibration sites are no more a constraint for the satellite daily programming.

\section{REFERENCES}

[1] Gachet R., "Caractérisation des Directions de Visée", Seminar "In-Orbit Geometric Characterization of Optical Imaging Systems" Bordeaux, 2-5 Nov 1999, SFPT bulletin $\mathrm{n}^{\circ} 159,(1999)$

[2] A. Bouillon, E. Breton, F. D. Lussy, and R. Gachet, "SPOT5 HRG and HRS first in-flight geometry quality results", in Proc. SPIE-Sensors, Systems, Next-Generation Satellites VI, 2003, vol. 4881, pp. 212-223.

[3] S. Sylvander, I. Albert-Grousset, P. Henry, "Geometrical Performances of the VEGETATION Products", IGARSS, Toulouse, Jul. 2003.

[4] Gachet R., "SPOT5 in-flight commissioning : inner orientation of HRG and HRS instruments", ISPRS Congress Istanbul, (2004)

[5] J-M. Delvit, P. Fave, R. Gachet, "The geometric supersite of Salon de Provence", ISPRS Congress Paris (2006)

[6] S. Leprince, P. Muse, and J. P. Avouac. "In-flight CCD Distortion Calibration for Pushbroom Satellites Based on Subpixel Correlation". IEEE Transactions on Geoscience and Remote Sensing, Vol.46, No.9, pp. 2675-2683, Sept. 2008.

[7] F. Ayoub, S. Leprince, R. Binet, K. Lewis, O. Aharonson and J. P. Avouac, "Influence of Camera Distortions on Satellite Image Registration and Change Detection Applications" in Proc. IGARSS, Boston, MA, USA, Jul. 2008.

[8] C. Latry, J-M. Delvit, "Staggered Arrays for high resolution earth observing systems", Earth Observing Systems XIV, proceedings of the SPIE, Volume 7452, pp. 74520O-74520O12, Jul. 2009.

[9] L. Lebegue, D. Greslou, F. Delussy, S. Fourest, C. Latry, P. Kubik, J-M. Delvit, "Pléiades-HR image quality commissioning foreseen methods", IGARSS, Honolulu, Jul. 2010.

[10] J-M. Delvit and al, "Geometric improvement for earth observation applications”, IGARSS, Honolulu, Jul. 2010.

[11] D. Greslou \& al, "Pleiades-HR innovative techniques for Geometric Image Quality Commissioning”, ISPRS Melbourne 2012.

[12] F. DeLussy \& al, "Pleiades HR in flight geometrical calibration : Localisation and mapping of the focal plane", ISPRS Melbourne 2012.

[13] A. Gleyzes \& al, "Pleiades system architecture and main performances", ISPRS Melbourne 2012.

[14] L. Lebegue \& al, "Pleiades-HR image quality commissioning”, ISPRS Melbourne 2012. 